\title{
WSN-based System in Computer Science for Monitoring Temperature outside Nuclear Reactor
}

\author{
Yiting Zhou ${ }^{1, \mathrm{a}}$, Sizong Shi ${ }^{1, \mathrm{~b}}$, Cunbo $\mathrm{Li}^{1}, \mathrm{Xin} \mathrm{Deng}^{1}, \mathrm{Bo} \mathrm{Su}^{1}$ \\ ${ }^{1}$ School of Information Engineering, Southwest University of Science and Technology, Mianyang \\ China \\ azhouyiting_2013@163.com, bshisizong@gd.swust.edu.cn
}

Keywords: wireless sensor network, routing protocol, humidity and temperature, monitor

\begin{abstract}
In this paper, a wireless sensor network is proposed as an approach to long-range communication in order to monitor data outside of the nuclear reactor. ATmega16 is used in the node to exercise master control; the net topology communication protocols and data collecting programs are developed with this node as the hardware platform. Experiments are carried out to monitor the humidity and temperature outside of the nuclear reactor. Experimental results show that the proposed system can accurately monitor the humidity and temperature changes and steadily display the humidity and temperature between nodes. Therefore, the proposed system can monitor the data outside the nuclear reactor as required.
\end{abstract}

\section{Introduction}

No component of the nuclear reactor monitoring system is more important than the centralized data collecting subsystem [1], because it is responsible for monitoring the operating status of the reactor in real-time. The humidity and temperature outside the reactor influence the security and reliability of the reactor devices. The data collected on humidity and temperature outside the reactor is extremely helpful for the nuclear power plant to monitor the operating status. Due to its low power consumption and mobility, the wireless sensor network (WSN) is a promising technique that can support the operation of the nuclear reactor if it is used for monitoring the data outside the reactor on a long-term basis [2]. WSN nodes on the Great Duck Island situated in Maine, U.S. are used to monitor the environmental data of the petrel habitat [3]. Mate Wel from Harvard and his team use WSN to continuously monitor the active volcano Tungurahua by sending the collected infrasonic wave data to the monitoring stations several myriameters away from the nodes [4].

Based on WSN, we employed net topology and Time Division Multiple Access (TDMA) to achieve multi-point communication, automatically collect and record data, issue fault alarms, fuse and analyze the data, and monitor the radiation remotely.

\section{Overall Structure of the System}

The entire data monitoring system is comprised of the controller and the measurer, which can communicate with each other via WSN that consists of the wireless data transmission module CC1101. The overall structure of the system is shown in Fig.1.

The structure of the system shows the system components, system working principles, and the direction in which the measurements flow. After being converted, the data is sent to the control processor in the measurer via the data bus, which then transmits, processes, and stores the data. The humidity and temperature measuring task is completed when the measurements are wirelessly delivered to the transceiver in the controller. To ensure data effectiveness, the controller needs to validate the received data according to the pre-determined protocol. After being processed, the data that is validated by the controller is stored and displayed, and alarms are issued if necessary. 


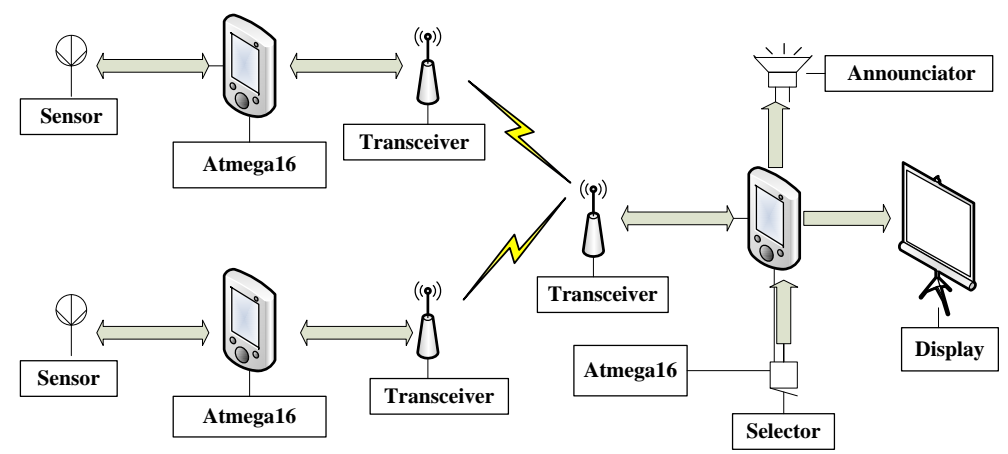

Fig.1. Structure of the data monitoring system

\section{Working Principles of the Measurer}

The measurer in the humidity and temperature measuring instrument mainly consists of the ATmega16 core processor, humidity and temperature sensors, the CC1101wireless communication module, the power conversion and voltage regulation module, the extended key set, LED status indicators, and the interface for subsequent upgrade. The measurer begins with measuring the two environmental variables (i.e. humidity and temperature) with the humidity and temperature sensors. The measurements are sent via SPI to the ATmega16 processor, and coded according to the communication protocol. The processed data is delivered to CC1101 using the serial ports and then transmitted as packets. When the measurer works normally, the corresponding status indicator shines; otherwise, the indicator is off.

\section{Working Principles of the Controller}

The controller in the humidity and temperature measuring instrument mainly consists of the ATmega16 core controlling system, the CC1101wireless communication module, the power conversion and voltage regulation module, the LCD display module, the human-computer interaction (HCI) key set, the alarm, the LED status module, and the interface for subsequent upgrade. First, the controller uses the CC1101 wireless module to receive the humidity and temperature measurements that are periodically sent by the measurer. Then, the ATmega16 core control system decodes and stores the received data and sends it to the 12864 LCD module for display. Typically, the LCD module is digital. When the user selects with the key, the LCD displays the curves. When the humidity and temperature measuring instrument works normally, the corresponding LED shines; otherwise, it is off. If the humidity and temperature measurements exceed the pre-determined value, then the buzzer issues the alarm for a period of time, until it is disabled or reaches the end of its alarm duration.

\section{WSN Communication Protocol Design}

WSN consists of many cheap small sensor nodes distributed throughout the detection area. It is a multi-hop, self-organizing communication network based on wireless communication, and its purpose is to collaboratively sense, collect, and process the information about the sensed objects within the network coverage area and send the data to the observer. The sensor, sensed object, and the observer constitute the key elements of WSN [5]. The proposed system has a master node and many secondary nodes, so it adopts the WSN net topology, as shown in Fig. 2.

The security of the humidity and temperature data does not need to be guaranteed during transmission in the proposed system. Instead, the main concern is the data quality and accuracy. Therefore, the measurements in the proposed system are not encrypted during transmission, however they need to undergo the data frame odd-even check and frame error detection. A unique ID is allocated to the node and the data serial numbers are added to the transmitted data to ensure accurate transmission of the data collected by the measurer, and alleviate data duplication and interference. 


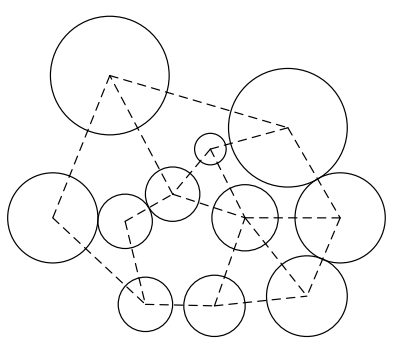

Fig.2.Net topology

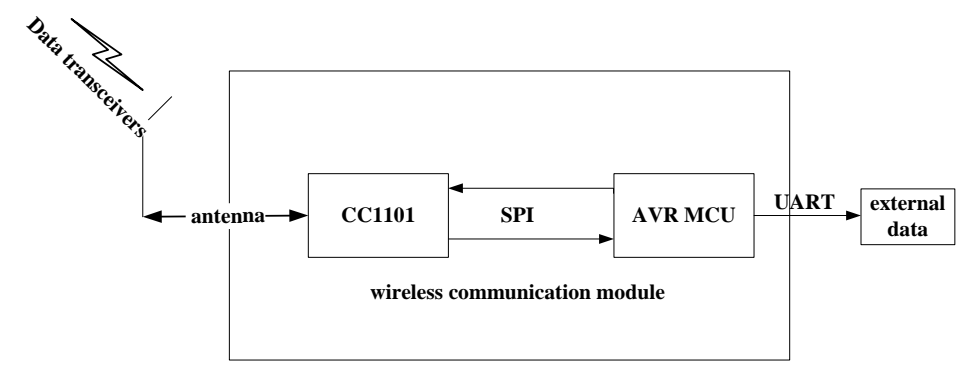

Fig.3. Data processing procedures

Communication protocol design.The communication protocol refers to the pre-determined agreement that the sender and the receiver must stick to during information exchange. By specifying all of the information that should be contained in the packets (e.g. packet size, data frame format, and check mode), the protocol helps to promote the accuracy and integrity of the data when it is sent and received, and also ensures that the data can be properly decoded from the received packets [6].

The communication between the controller and the measurer in the humidity and temperature measuring instrument is based on the CC1101 wireless module and its basic unit is the byte. The data packet format is $0 \times a a . . . .0 \times 55$, where $0 x a a$ is the data head, and $0 \times 55$ is the data tail. The size of the data packet is 10 bytes; the check mode is the odd check.

The first and second bytes correspond to the ID of the communicating device and the serial number of the transmitted packet, respectively. The 8-bit ID can recognize 256 devices at most, and each measurer has an ID. The 8-bit data packet serial number can recognize 256 packets at most. For packets that are transmitted erroneously and not received, the controller can rule out packets according to the serial numbers or request the measurer to retransmit the data.

The third and fourth bytes correspond to the high 8 bits and low 8 bits of the temperature data, which are the integer and the fractional part, respectively. When the temperature is negative, the top digit of the high 8 bits is set to 1 ; otherwise, it is set to 0 .

The fifth and sixth bytes correspond to the high 8 bits and low 8 bits of the humidity data, which are the integer and the fractional part, respectively.

The seventh and eighth bytes correspond to the 8-bit odd check and the 8 reserved bits. The 8-bit checksum is the sum of the $1^{\text {st }}, 2^{\text {nd }}, 3^{\text {rd }} 4^{\text {th, }} 5^{\text {th }}$, and $6^{\text {th }}$ bytes.

Procedures for processing wireless communication data.The wireless communication module consists of the CC1101 and AVR controller; it can forward data along with the antenna, as shown in Fig.3. The data received wirelessly by CC1101is first delivered to the controller via SPI. The controller then sends the data through the serial ports. Similarly, the data to be transmitted is first delivered to the controller through the serial ports, and the controller then sends the data to CC1101 via SPI for wireless transmission. If the controller and the wireless module are integrated with each other, then this wireless module can be used by any device that supports serial port.

\section{Setup of the Measuring System}

The measuring system is set up to ensure that the entire system can work normally. It is a comprehensive setup process that involves the collection of humidity and temperature data, CC1101 data transmission, data LCD display, and the ability to issue alarms and switch modes, i.e. the system needs to go through the entire humidity and temperature measuring procedure.

Actual tests show that the LCD displays the collected humidity and temperature normally. After pressing the mode switching key, the LCD displays the data curves. If the temperature exceeds the pre-determined temperature, the buzzer begins to make the buzzing sound. This demonstrates that the humidity and temperature measuring instrument is working normally. Fig. 4 and 5 provide the digital and pictorial description of the data, respectively. Fig. 6 shows the result of setting up the humidity and temperature measuring instrument on the LCD. 


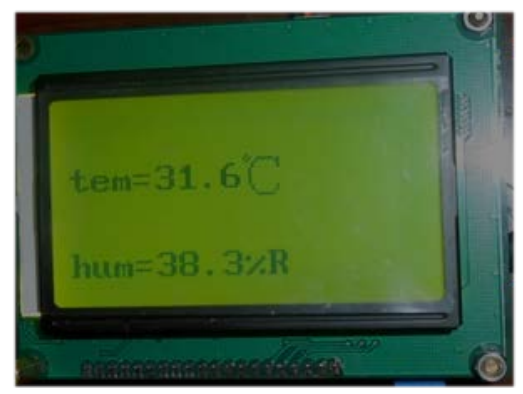

Fig. 4. Digital display of the data

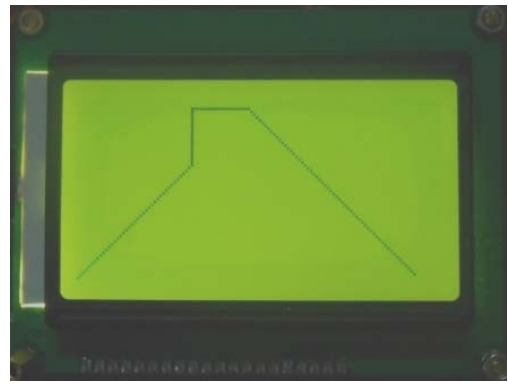

Fig.5.Curve display of the data

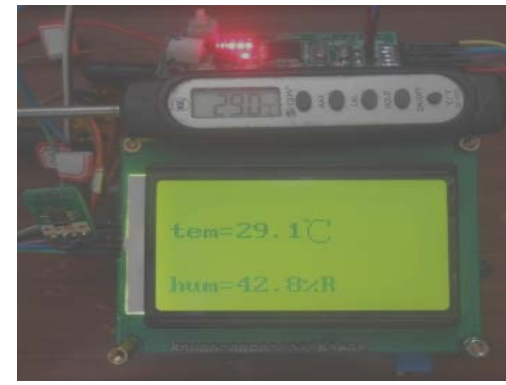

Fig. 6.result of setting up the measuring instrument

Fig. 4 and 5 show that we can randomly generate a set of data, store the data in the measuring instrument, digitally display it, and finally display the curve of the data. Fig.6 shows the data obtained by setting up the instrument many times; the data is almost the same as the measurements from the thermometer and the hygrometer, which shows that the temperature and humidity are being measured normally. During the test, it was observed that through the use of the Serial Assistant, the data can be returned, but it is fixed data that does not show any variations.

\section{Conclusions}

A wireless sensor network is proposed in this paper as an approach to long-range communication in order to monitor the data outside of the nuclear reactor. ATmega16 is used in the node to exercise master control; the net topology communication protocols and data collecting programs are developed with this node as the hardware platform. After comprehensive set-up and testing, the proposed system works reliability and meets the design needs. The power consumption and utility time are not taken into account in the proposed system. This design will be optimized in the future for better performance.

\section{Acknowledgments}

This work was supported in part by Science and Technology Department of Sichuan Province (2014RZ0049), This work was supported in part by Innovative talents of Science and Technology project in Sichuan committee (20132066).

\section{References}

[1]Rui Zhang, Huaqing Peng: Nuclear Power Engineering. Forum Vol. 23 (2002),P.86-88. (In Chinese)

[2]Zhihong Qian, Yijun Wang: Journal of Electronics and Information Technology. Forum Vol.35(2013),p.215-117. (In Chinese)

[3]Joseph Polastre, Robert Szewczyk, Alan Mainwaring, et al: Wireless sensor networks. Vol.35(2004),p.339-423.

[4] Werner-Allen J Johnson, Ruiz M, Lees J, et al in: Second European Workshop on Wireless Sensor Networks, IEEE, Istanbul,(2005).

[5]Li J, Wang F, in: IET International Communication Conference on Wireless Mobile and Computing , Shanghai(2011).

[6]Chao Lu: submitted to Journal of Materials Research (2011) . 